BONPLANDIA 18(1): 57-64. 2009

\title{
Novedades en Convolvuláceas argentinas
}

\author{
ANTONIO KRAPOVICKAS
}

Summary. Krapovickas, A. 2009. Novelties in Argentinean Convolvulaceae. Bonplandia 18(1): 57-64. ISSN: 0524-0476.

Three new species of Convolvulaceae from Argentina are described: Ipomoea Vivianae, Jacquemontia cataractae and J. estrellensis.

Key words. Ipomoea, Jacquemontia, taxonomy.

Resumen. Krapovickas, A. 2009. Novedades en Convolvuláceas argentinas. Bonplandia 18(1): 57-64. ISSN: 0524-0476.

Se describen cuatro especies nuevas de Convolvuláceas de Argentina: Ipomoea Vivianae, Jacquemontia cataractae y J. estrellensis.

Palabras clave. Ipomoea, Jacquemontia, taxonomía.

\section{Ipomoea Vivianae Krapov., sp. nov.}

Fig. 1

Perennis. Volubilis, $3 \mathrm{~m}$ alta, interdum decumbens. Ramuli cylindrici, pilis simplis, brevis. Petioli 3-4 cm eadem pubescentia quam caules instructa. Limbi subrotundati $4 \mathrm{~cm}$ longi, 3,5-4 cm lati; basi cordata, pubescentia sparsa. Cymae 10-20-florae. Pedunculi 3-5 cm longi. Bracteae $2 \mathrm{~mm}$ longae, caducae. Sepala exteriora pauce breviora, $8 \mathrm{~mm}$ longa, $5 \mathrm{~mm}$ lata. Corolla alba, tubo interne roseo, extus pilis paucis in parte superiore arearum mesopetalarum praedita. Capsulae subglobosae, $8 \mathrm{~mm}$ longae, $6 \mathrm{~mm}$ latae, glabratae, ochraceae, 2-locularae, 2-seminatae. Semina $5 \mathrm{~mm}$ longa, pilis 5-7 $\mathrm{mm}$ longis. Affinis Ipomoea Lilloana O'Donell sed carpidia minora, et sepala non retrorsae.

Holotypus: Argentina. Salta: Dep. Rivadavia,
Pluma de Pato, 2322'21"S, 6305’53"W, 237 m. En las vías férreas, suelo arenoso. Flores blancas. 13-II-2005. V. Solis Neffa, J.G. Seijo, J.G. Grabiele \& W. Reynoso 1985 (CTES). Isotypi: LIL, SI.

Voluble. Trepadora hasta $3 \mathrm{~m}$ de altura. Tallo cilíndrico, 2-5 mm de diámetro, liso, glabro o cubierto de pelos simples diminutos en las partes jóvenes. Pecíolo 3-4 cm long., con indumento similar al del tallo. Láminas suborbiculares, $4 \mathrm{~cm}$ long. x 3,5-4 cm lat., obtusas, base cordada, seno ancho, ambas caras con pelos simples muy pequeños, esparcidos. Cimas 10-20-floras. Pedúnculo 3-5 cm long., pedúnculos secundarios $1 \mathrm{~cm}$ long., pedicelos 1-1,5 cm long. Brácteas $2 \mathrm{~mm}$ long., caducas. Sépalos subiguales, los exteriores un poco más cortos, $8 \mathrm{~mm}$ long. x $5 \mathrm{~mm}$ lat., ápice subagudo, pubérulos, sépalos internos con el margen más claro. Corola blanca con el

${ }^{1}$ Instituto de Botánica del Nordeste, Casilla de Correo 209, 3400 Corrientes, Argentina. 


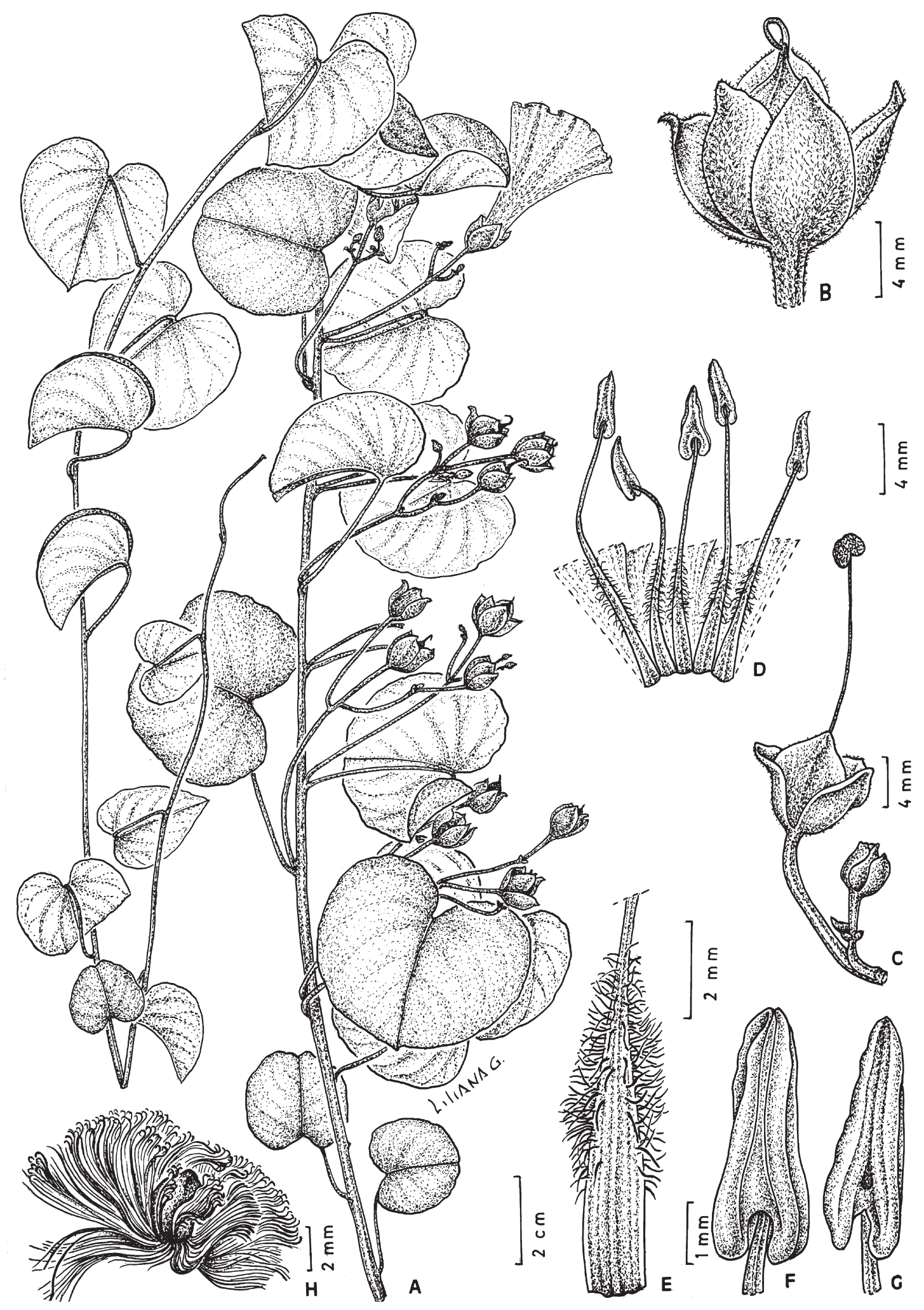

Fig. 1. Ipomoea Vivianae. A, rama; B, cáliz; C, gineceo; D, androceo; E, base del filamento; F, G, anteras; H, semilla. (A-H, Solis Neffa 1985). 
tubo rosado en el interior, exteriormente con pelos escasos en las áreas mesopétalas. Polen pantoporado, reticulado y con espinas 18 ìm long. Cápsulas $8 \mathrm{~mm}$ long. x $6 \mathrm{~mm}$ lat, glabras, ocráceas, terminadas en un apículo 2 mm long., 2-locular, 2-seminado. Semillas negras, $5 \mathrm{~mm}$ long., con pelos 5-7 mm long., abundantes.

Nombre vernáculo: 'hem' (Maranta \& Arenas 118), 'hemtaj' (Maranta, 1987:218, sub Ipomoea Lilloana).

Distribución geográfica: Vive principalmente en el chaco semiárido del norte de Argentina, $\mathrm{S}$ de Bolivia y del $\mathrm{W}$ de Paraguay. También crece en el $\mathrm{N}$ del Paraguay Oriental.

Paratypi: ARGENTINA. Formosa: Dep. Matacos, Ingeniero Juárez, 3-VI-1980, Pedersen 12909 (C, CTES). Salta: Dep. Rivadavia, 8 km E de Los Blancos, 8-IV-2004, Krapovickas \& al. 47903 (CTES); J. Solá (Morillo), Barrio Mataco, 8-I-1983, Maranta \& Arenas 118 (CTES). Coronel Juan Solá (Morillo), 10-XII-2005, Suárez 12 (CTES). BOLIVIA. Santa Cruz: Prov. Cordillera, Parque Nacional Kaa-Iya del Gran Chaco, 58 km W del campamento San José, 18²7’31"S 612'ㄷ'"W, 230 m, 13-VI-1998, Fuentes \& Navarro 2418 (CTES, USZ). PARAGUAY. Amambay: P.J. Caballero, 18-IV-1995, Schinini \& al. 29283 (CTES, FCQ); Parque Nacional Cerro Corá, Administración, 22-II-1994, Krapovickas \& Cristóbal 44938 (CTES); Colonia Lorito Picada, colindante S del Parque Nac. Cerro Corá, 22-II-1994, Krapovickas \& Cristóbal 44944 (CTES, FCQ, G, LIL, SP); 61 kn S de Bella Vista, 26-II-1994, Krapovickas \& Cristóbal 45042 (CTES, SP). Alto Paraguay: Mayor Pedro Lagerenza, 6045' W, 20 S, 16-IV-1978, Schinini \& Bordas 15091 (CTES). Boquerón: Mariscal Estigarribia, 11-V-1994, Krapovickas \& al. 45273 (CTES); Col. Fernheim, Filadelfia, IV-2000, August \& Ulmke 40 (CTES); id., V-2000, August \& Ulmke 48 (CTES); Colonia 4 de Mayo, 2254'51" S, 59,48’48" W, 27-V-1993, Mereles \& Degen 5148 (CTES, FCQ); Lolita, 50 km S del cruce Loma Plata con ruta Trans-Chaco, 27-II1991, Vanni \& al. 2426 (CTES); Ruta Transchaco, 41 km NW de Fortín Teniente E. Ochoa, 12-V-1994, Krapovickas \& al. 45295 (CTES).
Etimología: Especie dedicada a la Dra. Viviana Solis Neffa, quién coleccionó el ejemplar tipo.

Obs.1: Especie afín a Ipomoea Lilloana O’Donell, la cual se diferencia por sus frutos mayores (15-17 mm long. x 16-18 mm lat.) y por el cáliz reflexo a la madurez (O’Donell, 1959: 174, fig.18).

Obs. 2: Esta especie es utilizada por los indios wichi (matacos) del NE de Salta como planta acuífera, aprovechando sus órganos subterráneos suculentos (Arenas, 1993: 200, sub Ipomoea Lilloana).

\section{Jacquemontia cataractae Krapov., sp. nov.}

Fig. 2

Volubilis. Rami pilis tribracchiatis sparse vestiti. Petioli3-4 cm, pubescentes ut caules. Lamina ovata, usque ad $8 \mathrm{~cm}$ longa et $5 \mathrm{~cm}$ lata, basi subcordata, apice acuto, subtus et supra pilis tribracchiatus sparse. Cymae multiflorae. Pedunculi 3-17 cm longi, subglabri, pediceli 4-5 mm longi, glabri. Bracteae basali 2, lanceolatae, 14-20 mm longae et 2-4 $\mathrm{mm}$ latae, glabrae. Bracteae $8 \mathrm{~mm}$ longae et 1,5 $\mathrm{mm}$ latae, glabrae. Sepala exteriora $6 \mathrm{~mm}$ longa et 2,5 lata, acuta, margine ciliato; sepala interiora $4 \mathrm{~mm}$ longa 3 $\mathrm{mm}$ lata, obtusa, glabra. Corolla $20 \mathrm{~mm}$ longa, glabrata, caerulea vel lilacina. Stamina $7 \mathrm{~mm}$ longa; antherae sagittatae. Stigmatae 2, ovatae. Capsula sphaeroidea, $6 \mathrm{~mm}$ longa et 5,5 $\mathrm{mm}$ lata, glabra.

Holotypus: Argentina. Misiones: Dep. Iguazú, Parque Nacional Iguazú, Cataratas del Iguazú, voluble, flor celeste, 10-I-1970, A. Krapovickas \& C.L. Cristóbal 15608 (CTES).

Planta voluble. Tallo cilíndrico, con pelos de tres ramas, pequeños, esparcidos. Pecíolo 3-4 cm long., en el ápice de las ramas $0,5 \mathrm{~cm}$ long., pubescente. Lámina ovada, hasta $8 \mathrm{~cm}$ long. x $5 \mathrm{~cm}$ lat., base subcordada, ápice agudo, ambas caras con pelos de tres ramas esparcidos. Flores en cimas multifloras. 


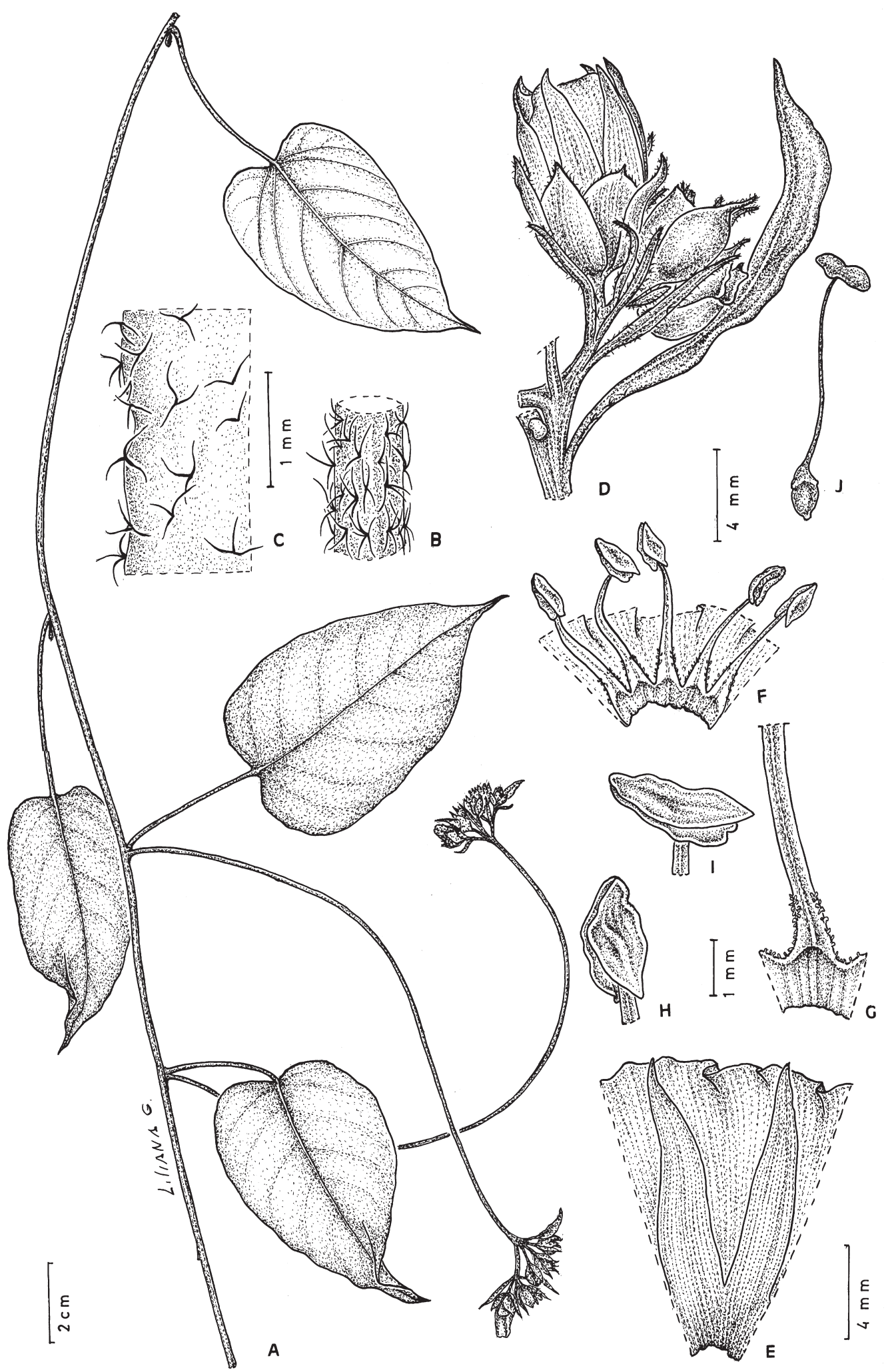

Fig. 2. Jacquemontia cataractae. A, rama; B. indumento del tallo; C,indumento del epifilo; D, inflorescencia; E, corola; F, androceo; G, base de filamento; H, I, anteras; J, gineceo. A-J, Krapovickas 15608. 
Pedúnculos 3-17 cm long., subglabros. Pedicelos 4-5 mm long., glabros. Brácteas basales 2, lanceoladas, 14-20 mm long. x 2-4 mm lat., glabras, oscuras. Brácteas florales 8 $\mathrm{mm}$ long. x 1,5 mm lat., glabras. Sépalos desiguales, los exteriores $6 \mathrm{~mm}$ long. x 2,5 mm lat., agudos, glabros, margen ciliado, los interiores algo menores, $4 \mathrm{~mm}$ long. $\mathrm{x} 3 \mathrm{~mm}$ lat., obtusos, mucronulados. Corola celeste, lila ó violácea, glabra, $20 \mathrm{~mm}$ long. Estambres 7 $\mathrm{mm}$ long, base de los filamentos con pelos glandulares, anteras sagitadas. Polen pantocolpado, pectado, perforado, con nanoespínulas. Estilo $10 \mathrm{~mm}$ long., estigmas 2, ovados, de base cóncava. Cápsula esférica, $6 \mathrm{~mm}$ long. x $5.5 \mathrm{~mm}$ lat., glabra.

Distribución geográfica: Vive en el Parque Nacional Iguazú, en las proximidades de las cataratas y en Paraguay, frente al río Paraná.

Paratypi: ARGENTINA. Misiones: Dep. Iguazú, Cataratas del Iguazú, 13-V-1951, Cabrera \& al. 62 (CTES, LP); id., 10-I-1970, Krapovickas \& Cristóbal 15608 (CTES); Parque Nacional del Iguazú, 7-I-2003, Garibaldi 169 (CTES); id., 19XII-1980, Cusato 486 (CTES, BAA); id., Sendero Yacaratiá inferior, 16-XII-1991, R. Vanni \& al. 2871 (CTES); id., sendero Macuco, 16-XII-1001, Placci 6 (CTES); id., sendero Barrio Guardaparques, $270 \mathrm{~m}$, 17-III-2002, Múlgura de Romero \& al. 3435 (CTES, SI); id., Ayo. El Ñandú, 17-XII-1993, Ahumada \& al. 7029 (CTES); id., Salto Arechea, 120 m, 2-XII1002, Vanni \& al. 3138 (CTES); id., Circuito Jacaratiá, 27-III-1996, Tressens \& al. 5592 (CTES); id., estación biológica, 24-XI-2003, A.A. Cocucci \& al. 3179 (CORD, CTES); id., Ruta prov. 101, camino al aeropuerto, 11-X-1996, Morrone \& al. 1185 (SI). PARAGUAY. Alto Paraná: Puerto Stroesner, 1IX-1983, N. Soria 235 (CTES, FCQ).

Etimología: Su nombre proviene de la localidad tipo, Cataratas del Iguazú.

Obs.: Especie muy parecida a J. Blanchetii Moric. por su aspecto general. Se diferencia porque en ésta las brácteas de la inflorescencia son muy pequeñas y no pasan de los 4-5 mm de longitud, y por el cáliz cuyos sépalos exteriores son menores que los interiores y obtusos.

\section{Jacquemontia estrellensis Krapov., sp. nov.}

Figs. 3 y 4

Volubilis, fusco-tomentosa, pili octobrachiati. Petiolo 10-20 $\mathrm{mm}$ longo. Lamina ovata vel subrotunda, usque ad $55 \mathrm{~mm}$ longa et $30 \mathrm{~mm}$ lata, margine integra supra et infra fusco-tomentosa. Cymae triflorae, pedunculo 5-10 $\mathrm{mm}$ longo. Pedicelo $1 \mathrm{~mm}$ longo. Bractea $19 \mathrm{~mm}$ longa $17 \mathrm{~mm}$ lata, cordata, tomentosa. Sepala exteriora $6-7 \mathrm{~mm}$ longa $2 \mathrm{~mm}$ lata, acuta; interiora $4 \mathrm{~mm}$ longa, acuta. Corolla caerulea, 10-17 mm longa, glabra. Stamina $5 \mathrm{~mm}$ longa. Stylus $6 \mathrm{~mm}$ longus, stigma 2-oblonga.

Holotypus: Argentina. Salta: Dep. Orán, 13 km E de Estrella, 3-V-1999, A. Krapovickas \& G. Seijo 47679 (CTES). Isotypi: LIL, NY, SP.

Voluble, toda la planta pardo-tomentosa, cubierta densamente de pelos estrellados pequeños, de 8 ramas. Pecíolo tomentoso, 10-20 mm long. Lámina oval a subcircular, ápice agudo a obtuso, hasta $55 \mathrm{~mm}$ long. x $30 \mathrm{~mm}$ lat., ambas caras fusco-tomentosas. Cimas trifloras, pedúnculo 5-10 mm long., fuscotomentoso. Pedicelos $1 \mathrm{~mm}$ long. Bráctea 19 mm long. x $17 \mathrm{~mm}$ lat., oval, base cordada, ápice acuminado, ambas caras tomentosas, pelos estrellados de 8 ramas. Sépalos externos 6-7 mm long. x $2 \mathrm{~mm}$ lat., sépalos internos 4 mm long., todos agudos. Corola azul a azul pálido, 10-17 mm long., glabra. Estambres 5, filamentos $5 \mathrm{~mm}$ long. Polen tricolpado. Estilo filiforme, $6 \mathrm{~mm}$ long., estigmas oblongos.

Distribución geográfica: Vive en el nordeste de Brasil, en la caatinga de Bahía y norte de Minas Gerais y en el chaco seco de Bolivia, Paraguay y del noroeste de Argentina en la provincia de Salta.

Paratypi: BOLIVIA. Cochabamba: Prov. Campero, bajada de Buena Vista al rio Grande, 1150 m, 27-I-1993, Antezana 622 (CTES). Santa Cruz: Prov. Cordillera, Ibasiri Izozog, la Brecha, 22-V-1999, Chávez de Michel 2682 (CTES, LPB).

BRASIL. Bahia: Juciape, 8-IV-1992, Hatschbach 56831 (CTES, MBM); 15 km S de 


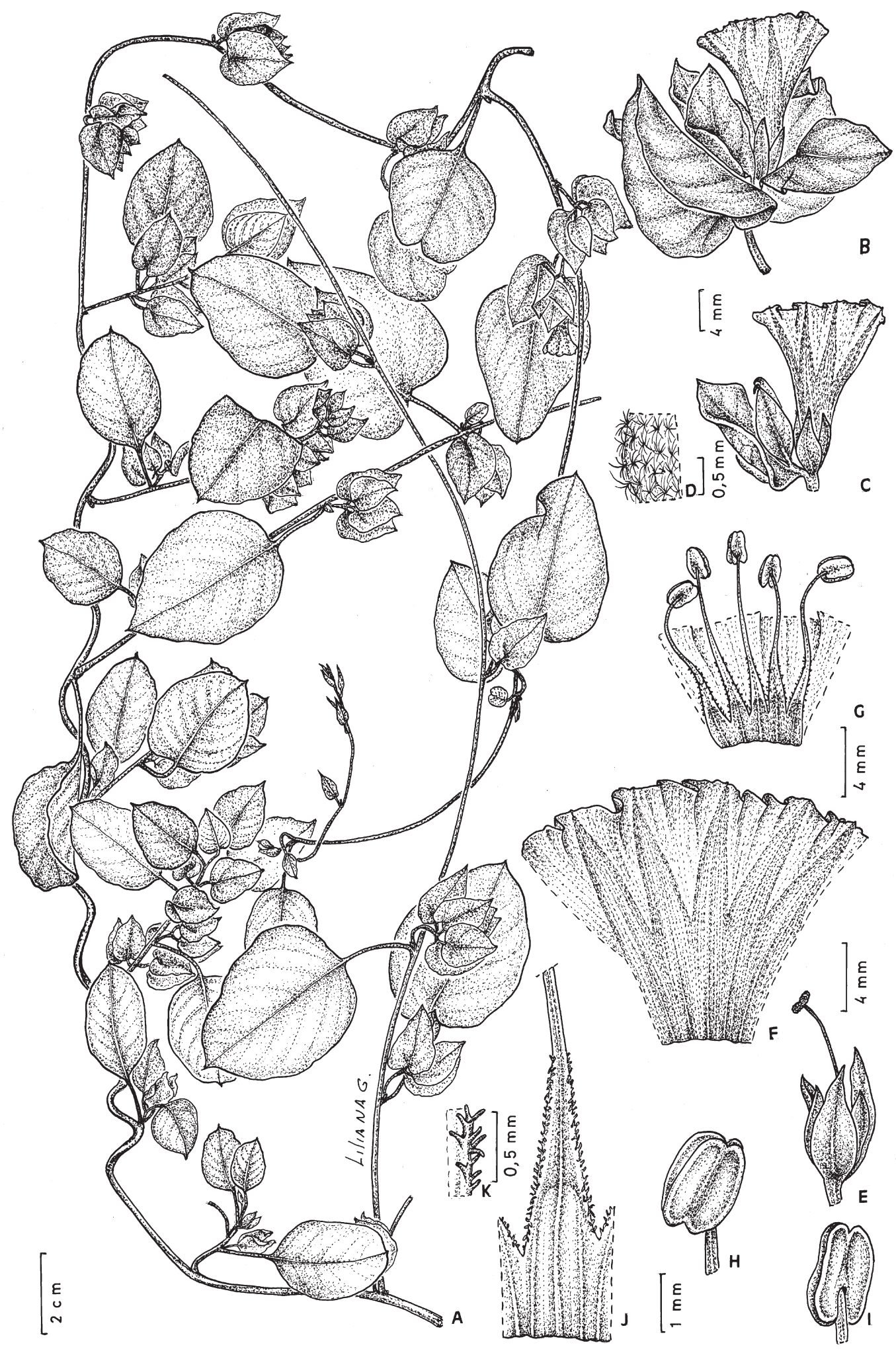

Fig. 3. Jacquemontia estrellensis. A, rama, B, inflorescencia; C, flor; D, indumento de la bráctea; E, estilo y estigma; F, corola; G, estambres; H, I, anteras; J, base de un estambre con detalle de los pelos. A-J, Krapovickas 47679. 

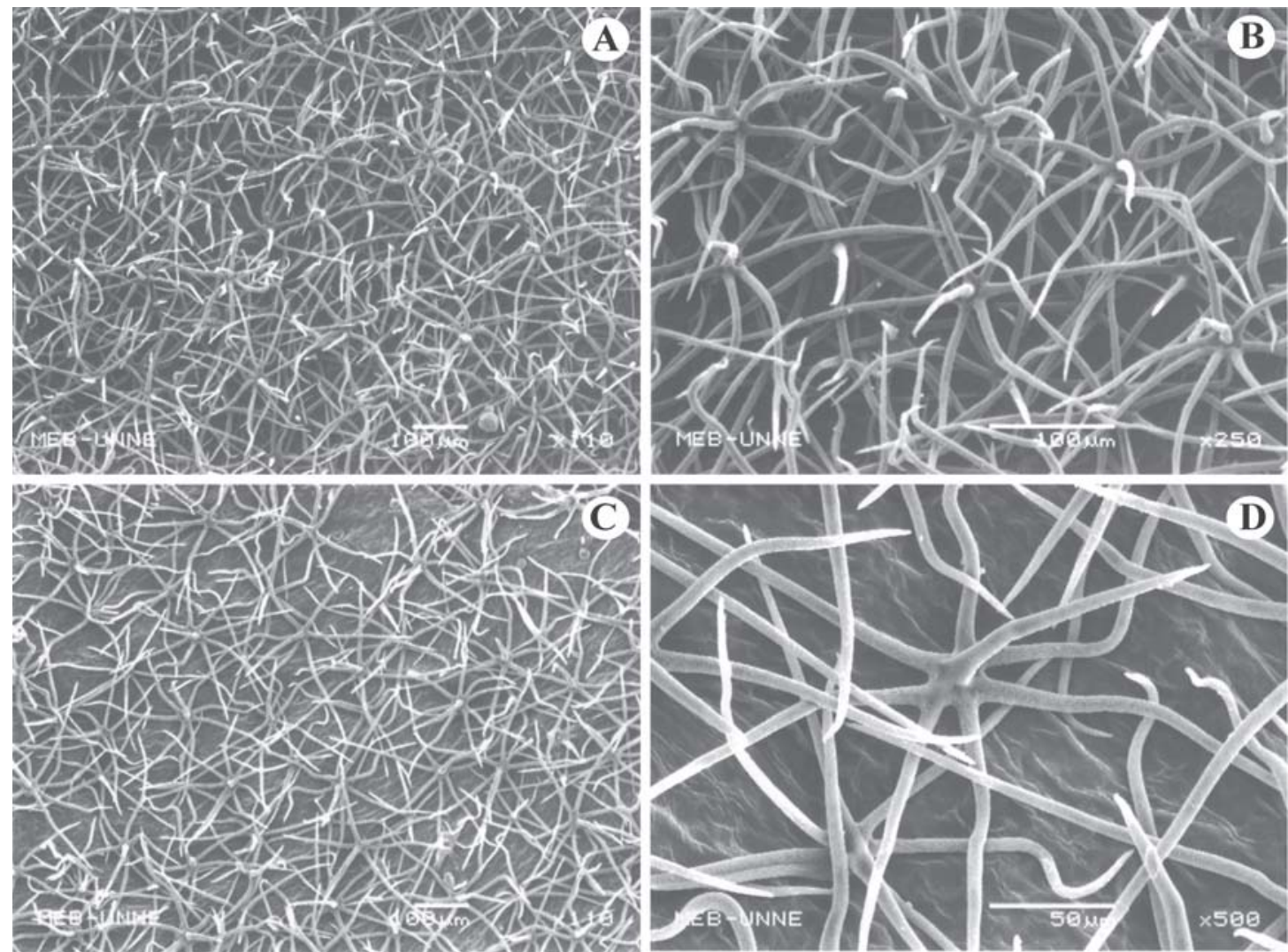

Fig. 4. Jacquemontia estrellensis, indumento de la bráctea. A, haz, x 110; B, haz, x 250; C, envés, x 110; D, envés, x 500. Todo de Hatschbach 64968.

Xique-Xique, camino a Santo Inácio, 1058’42"S, 4241’W, 400-500 m, 19-I-1997, Arbo \& al. 7515 $B$ (CEPEC, CTES); Mun. Barra, BR-242, 14-I1977, Hatschbach 39510 (CTES, MBM); Mun. Guanambi, BR-030, 10 km N de Guanambi, 900 m, 199-IV-1996, Hatschbach 65040 (CTES, MBM); Mun. Urandi, BR-122, proximo a divisa com Ouro Branco, 4-IV-1992, Hatschbach 56541 (CTES, MBM). Minas Gerais: Mun. Monte Azul, Serra do Espinhaço, subida via Montividiu, 19-IV1996, Hatschbach 64968 (CTES, LIL, MBM, SI). PARAGUAY. Alto Paraguay: Cerro León, 60¹5'W, 20²6'S, 30-IX-1979, Schinini \& Bordas 17812 (CTES).

Etimología: El nombre deriva de la localidad tipo, en la provincia de Salta, Argentina, lugar donde convergen elementos bolivianos.

Obs.: Las especies de Jacquemontia con sépalos externos cordiformes fueron ubicadas en la Flora Brasiliensis (Meissner, 1869) en el género Aniseia Choisy, "parviflorae", ellas son J. Velloziana (Mart.) O’Donell, J. gracillima (Choisy) Hallier f. y J. heterantha (Nees \& Mart.) Hallier f. (Austin, 1999). De todas ellas J. estrellensis se diferencia por el pedúnculo muy breve, que no sobrepasa la longitud del pecíolo. En cambio, las otras tienen pedúnculo igual o mayor que la hoja. Es más parecida a $J$. heterantha por la forma y el color de las hojas, pero se diferencia porque en ésta, además del pedúnculo 4-14 cm de largo, sus tricomas son (3)-4 radiados. La semejanza es aparente porque en J. estrellensis, la que tiene forma acorazonada es una bráctea y no los sépalos.

\section{Agradecimientos}

Agradezco a la Sra. Liliana Gómez la realización de los dibujos, al Dr. Pastor Arenas por 
la información sobre la utilización de los órganos subterráneos engrosados de Ipomoea Vivianae, por la etnia Wichi, y a la Lic. Cristina Salgado por los preparados de microscopía electrónica de barrido.

\section{Bibliografía}

ARENAS. P. \& G. C. GIBERTI. 1993. Etnobotánica de Jacaratia corumbensis O.Kuntze (Caricaceae) y reseña sobre otras plantas acuíferas del Gran Chaco.
Parodiana 8(2): 185-204.

AUSTIN, D. F. 1999. The genus Aniseia (Convolvulaceae). Systematic Botany 23(4): 411420.

MARANTA, A. A. 1987. Los recursos vegetales alimenticios en la etnia Mataco del Chaco centro occidental. Parodiana 5(1): 161-237.

MEISSNER, C. F. 1869. Convolvulaceae, in Mart. Fl.Brasil.7: 200-370.

O’DONELL, C. A. Convolvuláceas Argentinas. Lilloa 29: 87-348.

- 1960. Convolvuláceas II, Jacquemontia, Lilloa 30: 5-29.

Original recibido el 3 de junio de 2009; aceptado el 20 de agosto de 2009. 